\title{
Cheek and Periorbital Peculiar Discoid Lupus Erythematosus: Rare Clinical Presentation Mimicking Tinea Faciei, Cutaneous Granulomatous Disease or Blepharitis
}

\author{
Satoshi Nakamura Tomoko Yamada Naoka Umemoto \\ Toshinobu Nakamura Koji Wakatabi Eri Iida Masumi Masaki \\ Maki Kakurai Toshio Demitsu \\ Department of Dermatology, Saitama Medical Center, Jichi Medical University, Saitama, \\ Japan
}

\section{Key Words}

Discoid lupus erythematosus · Tinea faciei · Cutaneous granulomatous disease $\cdot$ Blepharitis

\begin{abstract}
We present clinically peculiar facial discoid lupus erythematosus (DLE) that mimicked tinea faciei. Although DLE is a chronic autoimmune dermatosis, it has a variety of rare clinical presentations, including periorbital DLE, comedonic DLE and hypertrophic DLE recently. In this case, a scaly, erythematous lesion on the eyelid and the central healed, mildly elevated, annularly distributed facial DLE mimicked tinea faciei, complicating our diagnosis.
\end{abstract}

(C) 2015 S. Karger AG, Basel

\section{Introduction}

We present clinically peculiar facial discoid lupus erythematosus (DLE) that mimicked tinea faciei, cutaneous granulomatous disease and blepharitis. Although DLE is a chronic autoimmune dermatosis, it has a variety of rare clinical presentations, including periorbital DLE [1], comedonic DLE [2] and hypertrophic DLE [3] recently. In this case, a scaly, erythematous lesion on the eyelid mimicked blepharitis. Moreover, the central healed, mildly 
elevated, annularly distributed facial DLE confused tinea faciei or granulomatous cutaneous disease [4]. Since a case of tinea faciei coexisting with DLE had previously been reported [5], this complicated our diagnosis.

\section{Case Presentation}

A 39-year-old Japanese man first noticed erythema on his right eyelid and right cheek in 2010. He received medical treatment (topical hydrocortisone butyrate propionate) at a local dermatological clinic. However, his symptoms did not improve, and laboratory testing at the neighborhood clinic revealed no abnormal findings. Therefore, he visited our hospital in 2012 to obtain a clinical examination and diagnosis for his cutaneous disease.

Clinical examination from the frontal view revealed eruptions on his bilateral inner and upper eyelids. The main signs of disease were well-demarcated, mildly elevated, darkred erythemas with pityriasis scales, and keratotic papules as well as pustules were also seen (fig. 1). In addition, swelling around the eyelids was visible, and these eyelid symptoms appeared to indicate blepharoconjunctivitis or allergic dermatitis. In addition, there were linearly distributed erythemas with lamellar scales, 1-cm dark-red erythemas with pityriatic scales and cutaneous very mild atrophy on the right side of his face. These eruptions had coalesced into one large annular, mildly elevated eruption, and pigmentation was visible at the center of the annular eruption, although no scales and erythemas were noticeable (fig. 2). There also appeared to be central healing and peripheral elevation as is observed in cases of tinea faciei or granulomas, especially granuloma trichophyticum, sarcoidosis and annular elastolytic giant cell granuloma. Hyphae were not observed after potassium hydroxide extraction, and his neck and cervical lymph nodes were not palpable.

Based on these findings, laboratory testing was performed, and a complete blood count revealed that his white blood cell count was slightly decreased to 2,180/ml (normal 3,000$7,800)$. His antinuclear antibody titers were slightly elevated to $40 \mathrm{U}$ (normal $<40$ ). Apart from that, the following laboratory results were normal: hemoglobin $10.5 \mathrm{~g} / \mathrm{dl}$ (normal 10.4-14.4), total protein 7.2 g/dl (normal 6.5-8.0), and albumin $4.7 \mathrm{~g} / \mathrm{dl}$ (normal 4.0-5.2). The results from liver function and renal function tests were also normal. His C-reactive protein level was $0.08 \mathrm{mg} / \mathrm{dl}$ (normal $<0.5$ ), and the results of tests for anti-double-strand DNA antigen, anti-SSA antigen, anti-SSB antigen and human immunodeficiency viral antibodies were also negative.

Therefore, we performed a skin biopsy of his right cheek, and cutaneous histopathological analysis with hematoxylin and eosin staining revealed parakeratosis and hyperkeratosis of the horny layer. Hydropic degeneration and vacuolar changes to the basal layer were also observed. However, in the dermis, typical dense lymphoid cells were observed around the follicles and eccrine glands (fig. 3). Mucin depositions were observed in the dermal stroma, and periodic acid-Schiff stain revealed that the basement membranes were thicker than those in normal skin. A direct immunofluorescence histopathology test revealed IgG deposition on the basement membrane zone and perifollicular areas (not shown). Based on these findings, we diagnosed his eruption as DLE. He used tacrolimus ointment, with good response. 


\section{Discussion}

From the lateral view, our case was clinically characterized by facial DLE that originally appeared to be tinea faciei or granulomatous cutaneous disease (fig. 2) [5-7]. Only one case of coexisting DLE and tinea faciei [5] and two cases of tinea faciei mimicking lupus erythematosus have been reported [6, 7]. A previous report also showed that DLE confused sarcoidosis, granuloma annulare and granuloma faciale [4]. In addition, our case exhibited bilateral eyelid involvement (fig. 1), and the eyelid DLE appeared to be blepharitis or allergic dermatitis. However, eyelid involvement is rare for DLE (only $6 \%$ of cases), and it is typically accompanied by other cutaneous eruptions [1]. Similar to our case, Gupta et al. [1] stated that the diagnosis of eyelid DLE can be significantly delayed due to difficulty in obtaining histopathology samples.

DLE has various uncommon subtypes [1-5], although the causes of these peculiar DLE subtypes are not clear [2]. The symptoms of facial DLE may mimic tinea faciei $[6,7]$ and other granulomatous cutaneous diseases [4]. Histopathologically, our case showed hyperkeratosis with parakeratosis, and no severe epidermal atrophy. The patient's mild facial atrophy was explained by this histopathology, and showed like tinea faciei. Additionally, the dermal severe dense lymphoid cell infiltrate around appendage might show partial mild elevation, resulted in granulomatous clinical appearance. These eruptions coalesced into one large annular, mildly elevated erythema also might mimic annular granulomatous appearance.

Our case reinforces the importance of considering the various clinical presentations of DLE, and dermatologists should be aware of these presentations, especially in cases with facial eruptions.

\section{Disclosure Statement}

The authors declare no conflicts of interest or financial support.

\section{References}

1 Gupta T, Beaconsfield M, Rose GE, Verity DH: Discoid lupus erythematosus of the periorbita: clinical dilemmas, diagnostic delays. Eye (Lond) 2012;26:609-612.

-2 Farias DF, Gondim RM, Redighieri IP, Muller H, Petri V: Comedonic lupus: a rare presentation of discoid lupus erythematosus. An Bras Dermatol 2011;86(4 suppl 1):S89-S91.

3 Narang T, Sharma M, Gulati N, Kaur A: Extensive hypertrophic lupus erythematosus: atypical presentation. Indian J Dermatol 2012;57:504.

4 Safer LF, Lang PG Jr, Demetree JW, Jones HE: Tinea faciei coexistent with discoid lupus erythematosus. Arch Dermatol 1981;117:121-122.

-5 Singh R, Bharu K, Ghazali W, Bharu K, Nor M, Kerian K: Tinea faciei mimicking lupus erythematosus. Cutis 1994;53:297-298.

-6 Meymandi S, Wiseman MC, Crawford RI: Tinea faciei mimicking cutaneous lupus erythematosus: a histopathologic case report. J Am Acad Dermatol 2003;48(2 suppl):S7-S8.

-7 Yaghoobi R, Feily A, Behrooz B, Yaghoobi E, Mokhtarzadeh S: Palpebral involvement as a presenting and sole manifestation of discoid lupus erythematosus. ScientificWorldJournal 2010;10:2130-2131. 


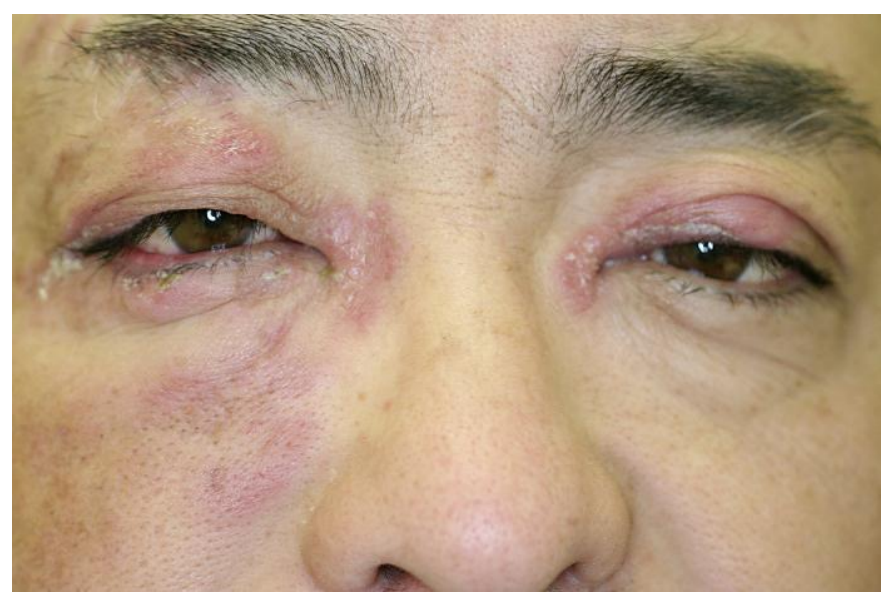

Fig. 1. Clinical presentation of the eyelid eruptions. Well-demarcated, dark-red, partially violet erythemas with small lamellar scales are visible on the patient's bilateral inner and upper eyelid from the frontal view. Mild eyelid edemas are also visible.

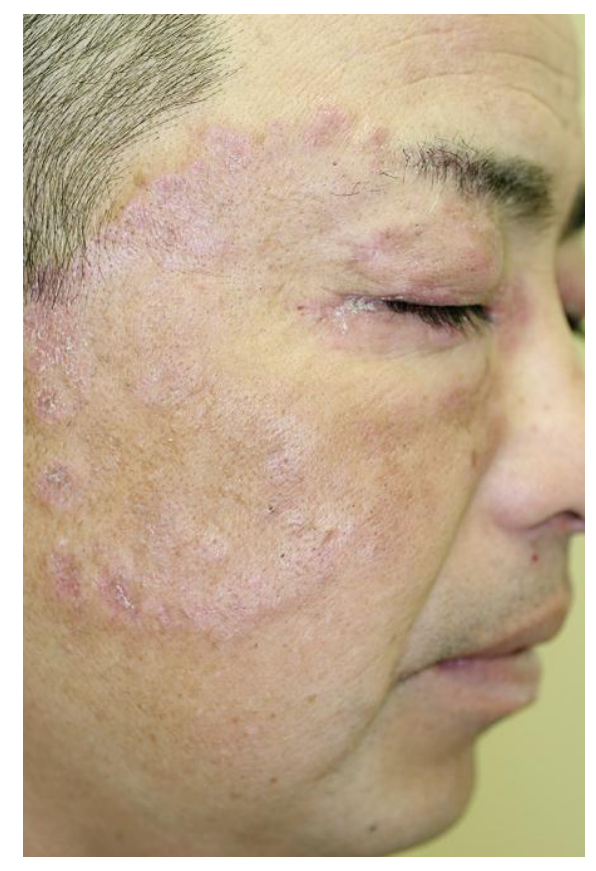

Fig. 2. Clinical presentation. A dark-red erythematous plaque (approximately $1 \mathrm{~cm}$ ) is accompanied by pityriatic scales, dark-red partially violet erythemas and coalesced atrophy, which form a large annular eruption on the right side of the patient's face. Pigmentation is visible at the center of the annular erythema, although the scales and erythemas have subsided. 
Nakamura et al.: Cheek and Periorbital Peculiar Discoid Lupus Erythematosus

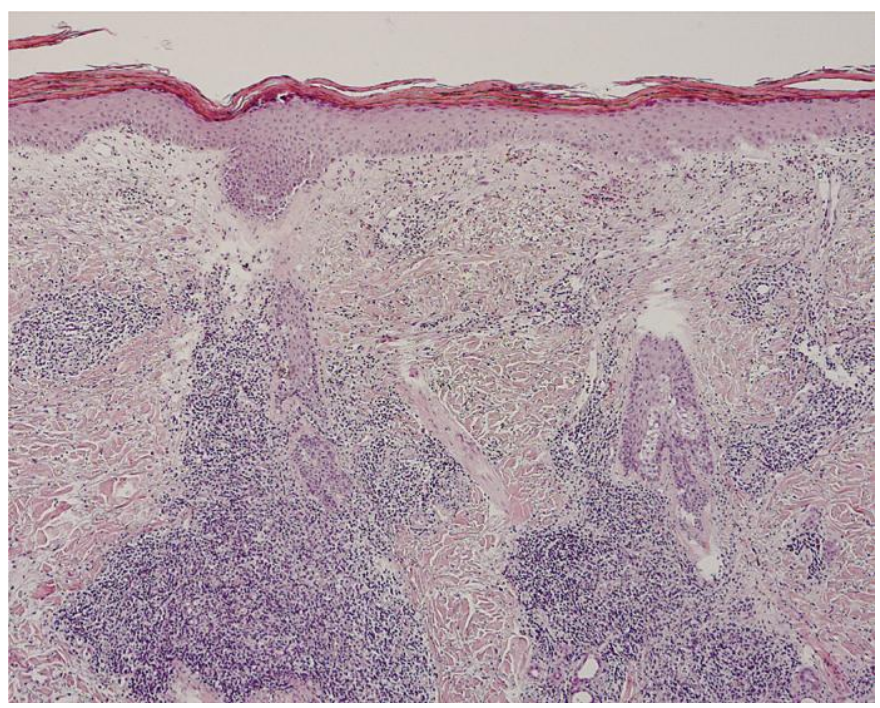

Fig. 3. Histopathological findings. Parakeratosis and hyperkeratosis of the horny skin layer as well as hydropic degenerations and vacuolar changes to the basal layer are visible. In the dermis, typical dense lymphoid cells are visible around the follicles or eccrine glands. Hematoxylin and eosin staining, $\times 40$. 